\title{
Array Representation of Entanglement for Pseudo-Quantum Gate Computing on Conventional Digital Circuits
}

This paper was downloaded from TechRxiv (https://www.techrxiv.org).

\section{LICENSE}

CC BY 4.0

SUBMISSION DATE / POSTED DATE

24-06-2020 / 25-06-2020

CITATION

Hirano, Takeshi (2020): Array Representation of Entanglement for Pseudo-Quantum Gate Computing on Conventional Digital Circuits. TechRxiv. Preprint. https://doi.org/10.36227/techrxiv.12554300.v1

$\mathrm{DOI}$

10.36227/techrxiv.12554300.v1 


\title{
Array Representation of Entanglement for Pseudo-Quantum Gate Computing on Conventional Digital Circuits
}

\author{
Hirano Takeshi *
}

$2020 / 06 / 24$

\begin{abstract}
Entanglement is important on quantum gate based computer. Simple implementation of simulated entanglement on conventional digital circuits requires spatial complexity $O\left(2^{N}\right)$ where $N$ is number of qubits. In this article, array representation of entanglement which requires spatial complexity $O\left(N^{2}\right)$ is proposed for simulated quantum gate on conventional digital circuits. Simulation shows proposed method can solve the problem which Deutsch-Jozsa algorithm solves.
\end{abstract}

\section{Background}

Entanglement is important on quantum gate based computer. Entanglement is caused by CNOT gate. XOR (namely CNOT) gate shrinks two input qubits to one output qubit. This output qubit has dependency to input qubits. Representation of qubit which consists of two complex number is not sufficient to represent this dependency. But representation based on divided cases which have complexity $O\left(2^{N}\right)$ seems to be too large. If this output dependent to $\mathrm{N}$ independent qubits, representation of the output should have information $O(N)$ not to lose any information with XOR. So I propose the representation of qubit which consists of array with length of $\mathrm{N}$.

\section{Proposed Representation}

Unitary gates and CNOT gates can make any other quantum gates. Investigating how unitary gates and CNOT gates affect to independent bits will help constructing the representation of its output.

Here we use independent qubits $q_{1}, q_{2}$, each of them consists of two complex number. $U$ represents $2 \times 2$ unitary matrix. NOT and $I$ are special case of $U$.

$$
\begin{aligned}
q_{1} & =\left(\begin{array}{l}
q_{1(0)} \\
q_{1(1)}
\end{array}\right) \\
q_{2} & =\left(\begin{array}{l}
q_{2(0)} \\
q_{2(1)}
\end{array}\right) \\
\text { NOT } & =\left(\begin{array}{ll}
0 & 1 \\
1 & 0
\end{array}\right) \\
I & =\left(\begin{array}{ll}
1 & 0 \\
0 & 1
\end{array}\right)
\end{aligned}
$$

$\left|q_{k(0)}\right|^{2}$ is the probability of that $k$ th qubit takes value 0 , as conventional representation of qubit.

When CNOT gate takes $q_{1}$ and $q_{2}$ as inputs, it outputs $q_{1}$ and $X O R\left(q_{1}, q_{2}\right)$. Combination of unitary gate and XOR gate behave like below.

$$
\begin{aligned}
\operatorname{XOR}\left(q_{1}, q_{2}\right) & =\left(q_{1(0)} \mid q_{1}=0>\right)\left(q_{2}\right)+\left(q_{1(1)} \mid q_{1}=1>\right)\left(\text { NOT. } q_{2}\right) \\
\operatorname{XOR}\left(q_{1}, U . q_{2}\right) & =\left(q_{1(0)} \mid q_{1}=0>\right)\left(U . q_{2}\right)+\left(q_{1(1)} \mid q_{1}=1>\right)\left(\text { NOT.U } q_{2}\right) \\
\text { U.XOR }\left(q_{1}, q_{2}\right) & =\left(q_{1(0)} \mid q_{1}=0>\right)\left(U . q_{2}\right)+\left(q_{1(1)} \mid q_{1}=1>\right)\left(\text { U.NOT. } q_{2}\right)
\end{aligned}
$$

From these behavior, two $2 \times 2$ matrices for one independent qubit seems to be required. Then output qubit $q_{o}$ which has dependency on independent $N$ qubits can be array like as Eq.8.

*hyranno4pub@gmail.com http://uncotechhack.net 


$$
q_{o}=\left\{M_{1[0]}, M_{1[1]}, M_{2[0]}, M_{2[1]}, \ldots M_{N[0]}, M_{N[1]}\right\}
$$

\subsection{Marginal probability}

To perform a measurement, probability of result 0,1 have to be calculated on output qubit $q_{o}$. From equations above, marginal probability $P_{m}$ of result on $q_{o}$ should be as Eq.11.

$$
\begin{aligned}
& f_{p}(M, q)=\left(\begin{array}{l}
\left|M_{(11)} q_{(0)}\right|^{2}+\left|M_{(12)} q_{(1)}\right|^{2} \\
\left|M_{(21)} q_{(0)}\right|^{2}+\left|M_{(22)} q_{(1)}\right|^{2}
\end{array}\right) \\
& f_{m p}(k)= \begin{cases}f_{p}\left(M_{k[0]}, q_{k}\right) & \text { if }(k=1) \\
f_{m p}(k-1)_{0} \cdot f_{p}\left(M_{k[0]}, q_{k}\right)+f_{m p}(k-1)_{1} \cdot f_{p}\left(M_{k[1]}, q_{k}\right) & \text { otherwise }\end{cases} \\
& P_{m}\left(q_{o}\right)=\quad f_{m p}(N)
\end{aligned}
$$

With this representation, $q_{o}$ may have $M_{k[0]}$ and $M_{k[1]}$ even if $q_{o}$ have no dependency on $q_{k}$, considering $C_{0}, C_{1}$ below.

$$
\begin{gathered}
C_{0}=\left(\begin{array}{ll}
1 & 1 \\
0 & 0
\end{array}\right) \\
C_{1}=\left(\begin{array}{ll}
0 & 0 \\
1 & 1
\end{array}\right) \\
\left(\begin{array}{l}
1 \\
0
\end{array}\right)=C_{0} \cdot q_{k} \\
\left(\begin{array}{l}
0 \\
1
\end{array}\right)=C_{1} \cdot q_{k}
\end{gathered}
$$

If $q_{o}$ has no dependency on $q_{k}$, then $M_{k[0]}=C_{0}$ and $M_{k[1]}=C_{1}$. So if $q_{o}=q_{j}$, $q_{o}$ can be represented as array like below.

$$
\begin{aligned}
& q_{o}=\left\{M_{1[0]}, M_{1[1]}, M_{2[0]}, M_{2[1]}, \ldots M_{N[0]}, M_{N[1]}\right\} \\
& M_{k[0]}= \begin{cases}I & \text { if }(k=j) \\
C_{0} & \text { otherwise }\end{cases} \\
& M_{k[1]}= \begin{cases}I & \text { if }(k=j) \\
C_{1} & \text { otherwise }\end{cases}
\end{aligned}
$$

While $P_{m}$ is marginal probability of single output, you need to calculate conditional probability when you measure multiple output. To calculate conditional probability, you will need $A N D\left(q_{o 1}, q_{o 2}\right)$.

\subsection{Unitary gate}

Assuming from Eq.7, unitary gate behave like below.

$$
\begin{aligned}
\left(M_{k[0]}, M_{k[1]}\right) \rightarrow & \begin{cases}\left(U \cdot M_{k[0]}, U \cdot M_{k[1]}\right) & \text { if }(k=m) \\
\left(M_{k[0]}, M_{k[1]}\right) & \text { otherwise }\end{cases} \\
& \text { where } m=\max \left(l \mid \operatorname{det}\left(M_{l[0]}\right) \neq 0\right)
\end{aligned}
$$

\section{$2.3 \quad$ CNOT gate}

I had little more investigation on XOR shown as Eq.21 and Eq.23. $E_{k(v)}$ and $f_{X O R}$ has been defined for shortening text as Eq.20 and Eq.22.

$$
E_{k(v)}=\left(q_{k(v)} \mid q_{k}=v>\right)
$$




$$
\begin{aligned}
& \operatorname{XOR}\left(M_{a} . q_{1}, M_{b} . q_{1}\right) \\
& =\left(\left(M_{a} \cdot q_{1}\right)_{(0)} \mid M_{a} \cdot q_{1}=0>\right) \cdot\left(M_{b} \cdot q_{1}\right)+\left(\left(M_{a} \cdot q_{1}\right)_{(1)} \mid M_{a} \cdot q_{1}=1>\right) \cdot\left(\text { NOT } \cdot M_{b} \cdot q_{1}\right) \\
& =\quad\left(\begin{array}{c}
M_{a(11)} \cdot M_{b(11)} E_{1(0)}+M_{a(12)} \cdot M_{b(12)} E_{1(1)} \\
M_{a(11)} \cdot M_{b(21)} E_{1(0)}+M_{a(12)} \cdot M_{b(22)} E_{1(1)}
\end{array}\right) \\
& +\left(\begin{array}{c}
M_{a(21)} \cdot M_{b(21)} E_{1(0)}+M_{a(22)} \cdot M_{b(22)} E_{1(1)} \\
M_{a(21)} \cdot M_{b(11)} E_{1(0)}+M_{a(22)} \cdot M_{b(12)} E_{1(1)}
\end{array}\right) \\
& =\left(\begin{array}{cc}
\left(M_{a(11)} \cdot M_{b(11)}\right)+\left(M_{a(21)} \cdot M_{b(21)}\right) & \left(M_{a(12)} \cdot M_{b(12)}\right)+\left(M_{a(22)} \cdot M_{b(22)}\right) \\
\left(M_{a(11)} \cdot M_{b(21)}\right)+\left(M_{a(21)} \cdot M_{b(11)}\right) & \left(M_{a(12)} \cdot M_{b(22)}\right)+\left(M_{a(22)} \cdot M_{b(12)}\right)
\end{array}\right) \cdot\left(q_{1}\right) \\
& f_{X O R}\left(M_{a}, M_{b}\right)=\left(\begin{array}{cc}
\left(M_{a(11)} \cdot M_{b(11)}\right)+\left(M_{a(21)} \cdot M_{b(21)}\right) & \left(M_{a(12)} \cdot M_{b(12)}\right)+\left(M_{a(22)} \cdot M_{b(22)}\right) \\
\left(M_{a(11)} \cdot M_{b(21)}\right)+\left(M_{a(21)} \cdot M_{b(11)}\right) & \left(M_{a(12)} \cdot M_{b(22)}\right)+\left(M_{a(22)} \cdot M_{b(12)}\right)
\end{array}\right)
\end{aligned}
$$

$$
\begin{aligned}
& \operatorname{XOR}\left(M_{a} \cdot \operatorname{XOR}\left(q_{1}, q_{2}\right), M_{b} \cdot q_{2}\right) \\
& =\quad X O R\left(M_{a} \cdot\left(\begin{array}{c}
E_{1(0)} E_{2(0)}+E_{1(1)} E_{2(1)} \\
E_{1(0)} E_{2(1)}+E_{1(1)} E_{2(0)}
\end{array}\right), M_{b} \cdot\left(\begin{array}{c}
E_{2(0)} \\
E_{2(1)}
\end{array}\right)\right) \\
& =\quad\left(M_{1(11)} \cdot\left(E_{1(0)} E_{2(1)}+E_{1(1)} E_{2(0)}\right)+M_{1(12)} \cdot\left(E_{1(0)} E_{2(0)}+E_{1(1)} E_{2(1)}\right)\right) \cdot\left(\begin{array}{c}
M_{2(11)} \cdot E_{2(0)}+M_{2(12)} \cdot E_{2(1)} \\
M_{2(21)} \cdot E_{2(0)}+M_{2(22)} \cdot E_{2(1)}
\end{array}\right) \\
& +\left(M_{1(21)} \cdot\left(E_{1(0)} E_{2(1)}+E_{1(1)} E_{2(0)}\right)+M_{1(22) \cdot} \cdot\left(E_{1(0)} E_{2(0)}+E_{1(1)} E_{2(1)}\right)\right) \cdot\left(\begin{array}{c}
M_{2(21)} \cdot E_{2(0)}+M_{2(22)} \cdot E_{2(1)} \\
M_{2(11)} \cdot E_{2(0)}+M_{2(12)} \cdot E_{2(1)}
\end{array}\right) \\
& =\quad\left(\begin{array}{l}
M_{1(11)} \cdot\left(M_{2(11)} \cdot E_{1(0)} E_{2(0)}+M_{2(12)} \cdot E_{1(1)} E_{2(1)}\right)+M_{1(12)} \cdot\left(M_{2(12)} \cdot E_{1(0)} E_{2(1)}+M_{2(11)} \cdot E_{1(1)} E_{2(0)}\right) \\
M_{1(11)} \cdot\left(M_{2(21)} \cdot E_{1(0)} E_{2(0)}+M_{2(22)} \cdot E_{1(1)} E_{2(1)}\right)+M_{1(12)} \cdot\left(M_{2(22)} \cdot E_{1(0)} E_{2(1)}+M_{2(21)} \cdot E_{1(1)} E_{2(0)}\right)
\end{array}\right) \\
& +\left(\begin{array}{l}
M_{1(21)} \cdot\left(M_{2(21)} \cdot E_{1(0)} E_{2(0)}+M_{2(22)} \cdot E_{1(1)} E_{2(1)}\right)+M_{1(22)} \cdot\left(M_{2(22)} \cdot E_{1(0)} E_{2(1)}+M_{2(21)} \cdot E_{1(1)} E_{2(0)}\right) \\
M_{1(21)} \cdot\left(M_{2(11)} \cdot E_{1(0)} E_{2(0)}+M_{2(12)} \cdot E_{1(1)} E_{2(1)}\right)+M_{1(22)} \cdot\left(M_{2(12)} \cdot E_{1(0)} E_{2(1)}+M_{2(11)} \cdot E_{1(1)} E_{2(0)}\right)
\end{array}\right) \\
& =\quad E_{1(0)} \cdot\left(\begin{array}{c}
\left(M_{1(11)} \cdot M_{2(11)}+M_{1(21)} \cdot M_{2(21)}\right) E_{2(0)}+\left(M_{1(12)} \cdot M_{2(12)}+M_{1(22)} \cdot M_{2(22)}\right) E_{2(1)} \\
\left(M_{1(11)} \cdot M_{2(21)}+M_{1(21)} \cdot M_{2(11)}\right) E_{2(0)}+\left(M_{1(12)} \cdot M_{2(22)}+M_{1(22)} \cdot M_{2(12)}\right) E_{2(1)}
\end{array}\right) \\
& +E_{1(1)} \cdot\left(\begin{array}{c}
\left(M_{1(12)} \cdot M_{2(11)}+M_{1(22)} \cdot M_{2(21)}\right) E_{2(0)}+\left(M_{1(11)} \cdot M_{2(12)}+M_{1(21)} \cdot M_{2(22)}\right) E_{2(1)} \\
\left(M_{1(12)} \cdot M_{2(21)}+M_{1(22)} \cdot M_{2(11)}\right) E_{2(0)}+\left(M_{1(11)} \cdot M_{2(22)}+M_{1(21)} \cdot M_{2(12)}\right) E_{2(1)}
\end{array}\right) \\
& =\quad E_{1(0)} \cdot\left(\begin{array}{cc}
\left(M_{1(11)} \cdot M_{2(11)}+M_{1(21)} \cdot M_{2(21)}\right) & \left(M_{1(12)} \cdot M_{2(12)}+M_{1(22)} \cdot M_{2(22)}\right) \\
\left(M_{1(11)} \cdot M_{2(21)}+M_{1(21)} \cdot M_{2(11)}\right) & \left(M_{1(12)} \cdot M_{2(22)}+M_{1(22)} \cdot M_{2(12)}\right)
\end{array}\right) \cdot\left(q_{2}\right) \\
& +E_{1(1)} \cdot\left(\begin{array}{ccc}
\left(M_{1(12)} \cdot M_{2(11)}+M_{1(22)} \cdot M_{2(21)}\right) & \left(M_{1(11)} \cdot M_{2(12)}+M_{1(21)} \cdot M_{2(22)}\right) \\
\left(M_{1(12)} \cdot M_{2(21)}+M_{1(22)} \cdot M_{2(11)}\right) & \left(M_{1(11)} \cdot M_{2(22)}+M_{1(21)} \cdot M_{2(12)}\right)
\end{array}\right) \cdot\left(q_{2}\right) \\
& =\quad E_{1(0)} \cdot f_{X O R}\left(M_{a}, M_{b}\right) \cdot\left(q_{2}\right)+E_{1(1)} \cdot f_{X O R}\left(M_{a} \cdot N O T, M_{b}\right) \cdot\left(q_{2}\right)
\end{aligned}
$$

Assuming from Eq.23, CNOT gate behave like below.

$$
\left(\left(M_{a k[0]}, M_{a k[1]}\right),\left(M_{b k[0]}, M_{b k[1]}\right)\right) \rightarrow\left(f_{X O R}\left(M_{a k[0]}, M_{b k[0]}\right), f_{X O R}\left(M_{a k[1]}, M_{b k[1]}\right)\right)
$$

\section{Simulation}

With Eq.11, Eq.19 and Eq.24, quantum gate computing should be simulated. But I have not got correct mathematical proof for this representation yet. So I wrote a simulation to show it work fine. Simulation is written in SystemVerilog and run by ModelSim. SystemVerilog has been chosen to ensure this representation can be implemented as physical gates, not only software.

\subsection{Problem}

This simulation solves the problem which Deutsch-Jozsa algorithm solves. In this problem, we are given a black box quantum computer as an oracle, which implements a function $f:\{0,1\}^{N} \rightarrow\{0,1\}$. This function is either "constant" or "balanced". If it is constant, the function returns always 0 , or always 1 . If it is balanced, the function returns 0 for half of the input domain and 1 for the others. The task is to determine if the $f$ is constant or balanced by using the oracle. In this simulation, also the oracle uses proposed array representation. 


\subsection{Implementation}

You can take a look and run the attached SystemVerilog code for detail. Unlike real-quantum computer, we can get marginal probability of the output. So what we have to do is to initialize to 0 and apply Hadamard gate to each input qubit, then connect all to the oracle and calculate the marginal probability of the output. If the probability is $\{1,0\}$, the function is constant returning 0 . If the probability is $\{0,1\}$, the function is constant returning 1 . If the probability is $\{0.5,0.5\}$, the function is balanced.

\subsection{Result}

Simulation works fine for number of qubits 4, solving the problem correctly.

\section{Conclusion}

Array representation of entanglement is proposed. With this proposed method, we can at least solve the Deutsch-Jozsa problem on conventional circuits in spatial complexity $O\left(N^{2}\right)$ instead of $O\left(2^{N}\right)$, where $N$ is number of input qubits. 